\title{
Microscopic Nature of the First-Order Field-Induced Phase Transition in the Strongly Anisotropic Ferrimagnet $\mathrm{HoFe}_{5} \mathrm{Al}_{7}$
}

\author{
D. I. Gorbunov, ${ }^{1}$ C. Strohm, ${ }^{2}$ M. S. Henriques, ${ }^{3,4}$ P. van der Linden, ${ }^{5}$ B. Pedersen, ${ }^{6}$ N. V. Mushnikov, ${ }^{7}$ \\ E. V. Rosenfeld, ${ }^{7}$ V. Petř́čcek, ${ }^{4}$ O. Mathon, ${ }^{5}$ J. Wosnitza, ${ }^{1,8}$ and A. V. Andreev ${ }^{4}$ \\ ${ }^{1}$ Hochfeld-Magnetlabor Dresden (HLD-EMFL), Helmholtz-Zentrum Dresden-Rossendorf, 01328 Dresden, Germany \\ ${ }^{2}$ Deutsches Elektronen-Synchrotron DESY, 22607 Hamburg, Germany \\ ${ }^{3}$ Institut Laue Langevin, 71 Avenue des Martyrs, F-38042 Grenoble, France \\ ${ }^{4}$ Institute of Physics, Academy of Sciences, Na Slovance 2, 18221 Prague, Czech Republic \\ ${ }^{5}$ European Synchrotron Radiation Facility, B.P. 220, 38043 Grenoble, France \\ ${ }^{6}$ Heinz Maier-Leibnitz Zentrum (MLZ), Technische Universität München, Lichtenbergstrasse 1, 85748 Garching, Germany \\ ${ }^{7}$ Institute of Metal Physics, Ural Branch of the Russian Academy of Sciences, Kovalevskaya 18, 620990 Ekaterinburg, Russia \\ ${ }^{8}$ Institut für Festkörperphysik, TU Dresden, D-01062 Dresden, Germany
}

(Received 8 September 2017; revised manuscript received 8 October 2018; published 28 March 2019)

\begin{abstract}
We report on X-ray magnetic circular dichroism experiments in pulsed fields up to $30 \mathrm{~T}$ to follow the rotations of individual magnetic moments through the field-induced phase transition in the ferrimagnet $\mathrm{HoFe}_{5} \mathrm{Al}_{7}$. Near the ground state, we observe simultaneous stepwise rotations of the Ho and Fe moments and explain them using a two-sublattice model for an anisotropic ferrimagnet with weak intersublattice exchange interactions. Near the compensation point, we find two phase transitions. The additional magnetization jump reflects the fact that the Ho moment is no longer rigid as the applied field acts against the intersublattice exchange field.
\end{abstract}

DOI: 10.1103/PhysRevLett.122.127205

Hard magnetic materials have many technical applications. Historically, rare-earth $(R)$ transition-metal $(T)$ intermetallics quickly superseded steels, alnico alloys, and ferrites due to the possibility to combine the high magnetic ordering temperatures typical of the $3 d$ metals with the large magnetic anisotropies of the magnetic rare earths. The interaction between the itinerant $3 d$ and localized $4 f$ electrons proceeds through the interatomic $3 d-5 d$ exchange and the intraatomic $5 d-4 f$ exchange [1]. In a given compound, the $3 d-4 f$ interaction transfers the strong exchange interactions from the $T$ to the $R$ sublattice and the large magnetocrystalline anisotropy from the $R$ to the $T$ sublattice [2]. Understanding the intrinsic magnetic properties is, thus, of fundamental and practical importance.

Application of an external magnetic field to single-crystal samples allows one to finely tune the exchange and anisotropy interactions and extract magnetic parameters by revealing particularities in the magnetization process [3-8]. Ferrimagnetic compounds having antiparallel coupling between the $3 d$ and $4 f$ magnetic moments can be studied most effectively by magnetization measurements since the ferrimagnetic structure is affected by the applied field providing direct information on the intersublattice exchange interaction and magnetic anisotropy. The use of this approach is, however, much less straightforward when the magnetization process is complicated by a pronounced field-induced noncollinearity of magnetic moments [9]. A detailed understanding requires a microscopic technique for the determination of the zero-field magnetic structure and its evolution in the applied field. Yet, for the hard magnetic materials, it is exactly the strength of the interactions, desirable for applications, that makes such a study challenging due to the large exchange and anisotropy energy.

$\mathrm{HoFe}_{5} \mathrm{Al}_{7}$ is a strongly anisotropic ferrimagnet whose $3 d-4 f$ exchange interactions can be broken by the application of magnetic fields accessible to some microscopic techniques [10]. At $2 \mathrm{~K}$, two field-induced magnetic phase transitions are observed at 13-20 and 34-38 T. Here, we combine neutron scattering in zero magnetic field for the determination of the moments and magnetic structure with $\mathrm{x}$-ray magnetic circular dichroism (XMCD) in pulsed magnetic fields to follow the individual sublattice magnetizations up to $30 \mathrm{~T}$. We observe rotations of the Ho and Fe magnetic moments across the first-order field-induced magnetic phase transition below and above the compensation point and estimate the anisotropy constant close to the ground state by employing a model for an anisotropic two-sublattice ferrimagnet with weak intersublattice exchange interactions. We also find that above the compensation point an approximation of nonrigid magnetic sublattices provides a more accurate description of the field-dependent magnetization.

$\mathrm{HoFe}_{5} \mathrm{Al}_{7}$ crystallizes in the tetragonal crystal structure of $\mathrm{ThMn}_{12}$ type. It orders ferrimagnetically at the Curie temperature of $T_{C}=216 \mathrm{~K}$ and has a compensation point of $T_{\text {comp }}=65 \mathrm{~K}$ [10]. The magnetic moments lie in the basal plane, the [110] axis is the easy magnetization 
direction (EMD). It was proposed that the two fieldinduced phase transitions observed along the EMD involve simultaneous rotations of the $\mathrm{Fe}$ and $\mathrm{Ho}$ magnetic moments, although a microscopic understanding is still lacking.

Results of powder neutron-diffraction experiments for $\mathrm{HoFe}_{5} \mathrm{Al}_{7}$ were reported in Ref. [11], where the data for $\mathrm{HoFe}_{5} \mathrm{Al}_{7}$ were accidentally interchanged with those of another compound [12]. This further motivates us to reexamine the magnetic structure of $\mathrm{HoFe}_{5} \mathrm{Al}_{7}$ in zero field. A neutron-diffraction study was done at the thermal single-crystal diffractometer RESI at FRM-II (Garching, Germany) using a wavelength of $\lambda=1.0433 \AA$ [14]. 2D datasets were collected at 2, 65, 165, and $300 \mathrm{~K}$. Structural refinements with JANA2006 [15] of the room-temperature data have shown that the structure of $\mathrm{HoFe}_{5} \mathrm{Al}_{7}$ is well described by the tetragonal $\mathrm{ThMn}_{12}$ type (space group $I 4 / \mathrm{mmm}$ ), in agreement with previous work [16]. In particular, for our single crystal, it was found that all crystallographic positions in the unit cell are fully occupied, except for the $8 j$ site, which has mixed occupancy by $\mathrm{Fe}(25 \%)$ and $\mathrm{Al}(75 \%)$.

Below the Curie temperature, magnetic scattering is observed at the positions of the nuclear Bragg peaks. Thus, the long-range magnetic order is characterized by a $\boldsymbol{k}=0$ wave propagation vector. A symmetry analysis has shown that from the $12 \boldsymbol{k}$-maximal space groups of $I 4 / \mathrm{mmm}^{\prime}$, only the magnetic Shubnikov group $F \mathrm{~mm}^{\prime} \mathrm{m}^{\prime}$ allows a global ferrimagnetic structure for $\mathrm{HoFe}_{5} \mathrm{Al}_{7}$ [17] consistent with previous macroscopic measurements. Specifically, within the Ho and Fe sublattices, the spins are ferromagnetically ordered in a collinear arrangement confined to the $a b$ plane, being antiparallel to each other (inset of Fig. 1). All Fe spins in the $8 j$ sites have the same orientation as those in the $8 f$ sites. Nevertheless, the $8 f$ position is split in two, Fe1_1 and Fe1_2, due to the symmetry reduction triggered by the magnetic ordering, with the three distinct spins, Fe1_1, $\mathrm{Fe} 1 \_2$, and Fe2, having different magnitudes of their magnetic moments (inset of Fig. 1 and Table I in the Supplemental Material [18]). In addition, extinction rules [22] and intensities of the reflections of the type $(00 l)$ with $l=2 n$ and $(h k l)$ with $h, k=2 n+1$ and $l=2 n$ (Table $\mathrm{I}$ in the Supplemental Material [18]) are in agreement with the description of the magnetic structure of both sublattices ordered and with the moments restricted to the $a b$ plane, which is kept below $T_{C}$. This is reasonably in line with the temperature dependence of the magnetization calculated using mean-field theory (see the Supplemental Material [18]) as shown in Fig. 1.

At $2 \mathrm{~K}$, the total magnetic moment of the $\mathrm{Fe}$ atoms extracted from our neutron data is $M=-6.3(5) \mu_{B} /$ f.u., whereas the Ho magnetic moment is $M=9.2(3) \mu_{B} /$ f.u., with the total (spontaneous) magnetization being $M_{s}=2.9(8) \mu_{B} /$ f.u. The moments of both sublattices

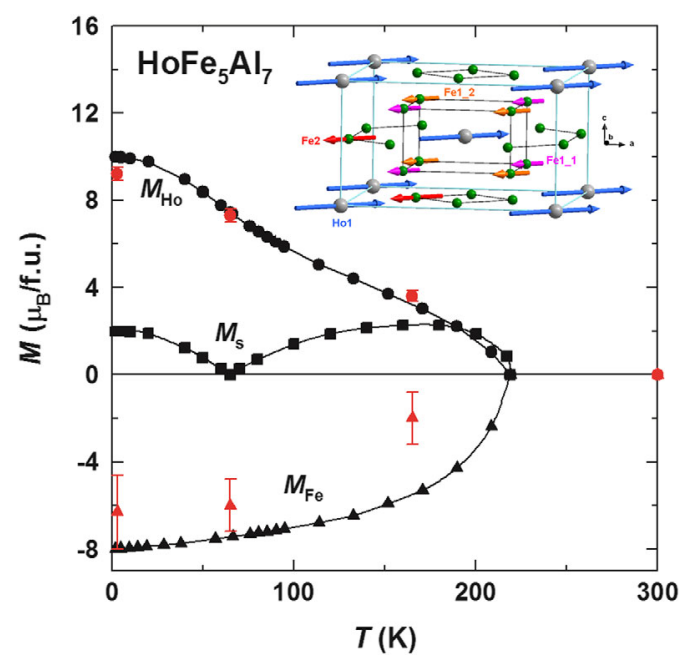

FIG. 1. Temperature dependence of the spontaneous magnetic moment, $M_{s}$, determined from bulk magnetization measurements (square symbols) and of the total magnetic moments of the Ho and Fe sublattices, $M_{\mathrm{Ho}}$ and $M_{\mathrm{Fe}}$, respectively, calculated within the mean-field approach (black symbols; see the Supplemental Material [18]), and determined from the neutron-scattering data (red symbols) for $\mathrm{HoFe}_{5} \mathrm{Al}_{7}$. (Inset) The magnetic structure of $\mathrm{HoFe}_{5} \mathrm{Al}_{7}$ at $2 \mathrm{~K}$. Only the magnetic atoms are depicted and the Ho magnetic moment is reduced twice so that the Fe moments can be clearly seen.

decrease as the temperature increases to the compensation point. At $165 \mathrm{~K}$, the atom Fe1_2 seems not to carry an ordered magnetic moment anymore.

Finally, it is important to bear in mind that the index of the orthorhombic magnetic space group, $F \mathrm{~mm}^{\prime} \mathrm{m}^{\prime}$, is 4 . As the coset representatives in the decomposition of the tetragonal magnetic space group with respect to the orthorhombic subgroup have a fourfold axis and time inversion, there are two equivalent nontrivial twinned magnetic configurations related by the fourfold rotation. The magnetic structure of $\mathrm{HoFe}_{5} \mathrm{Al}_{7}$ is also compatible with an orthorhombic distortion of the lattice, although to the best of our knowledge no such distortion has been observed in zero field.

Besides neutron diffraction, we have performed XMCD measurements at the $\mathrm{Fe} K$ edge (this Letter) and Ho $L_{2,3}$ edges (unpublished) of $\mathrm{HoFe}_{5} \mathrm{Al}_{7}$, with the goal to probe the $\mathrm{Fe}$ - and Ho-sublattice magnetizations as a function of field applied along the [110] axis above and below the compensation temperature. In order to reach the first critical field observed in bulk magnetization measurements [10], a miniature pulsed magnet system [23] reaching $30 \mathrm{~T}$ with a pulse duration of $\approx 1 \mathrm{~ms}$ was employed. XMCD experiments were carried out in transmission at the energy dispersive $\mathrm{x}$-ray absorption spectroscopy beam line ID24 at the ESRF [24]. A multiframe acquisition scheme was used to record energy spectra over the entire field pulse [25].

Samples were oriented along the [110] axis, cut, and polished to thin platelets. The latter were mounted on 
substrates of vitreous carbon, which are placed directly in the He flow of the sample cryostat.

As a function of field and temperature, the XMCD spectra at all three edges vary in both amplitude and shape, defeating a direct interpretation of spectral amplitudes and integrals. Similar observations were made in previous XMCD studies of other $R-T$ intermetallic systems [2632]. Systematic investigations have revealed that the variation of the spectral shape is a consequence of a mutually induced signal arising from the other magnetic constituent and transferred through the $R(5 d)-T(3 d)$ hybridization. It was shown that the induced signal at the $T$ site is proportional to the magnetization of the $R$ system [26-28,32] and vice versa [29-32]. This was exploited to probe the magnetization of both constituents at a single absorption edge [32]. Different methods were devised to disentangle these contributions [28,32]. Here, we applied singular value decomposition (SVD) to the XMCD spectra [33] to show that the data are indeed described by an orthogonal base of two dominating spectral components. This base obtained through SVD is defined except for a rotation in the plane it spans. We therefore used bulk magnetization data and neutron-scattering data in zero field to determine the base rotation and identify the spectral components representing the iron and holmium
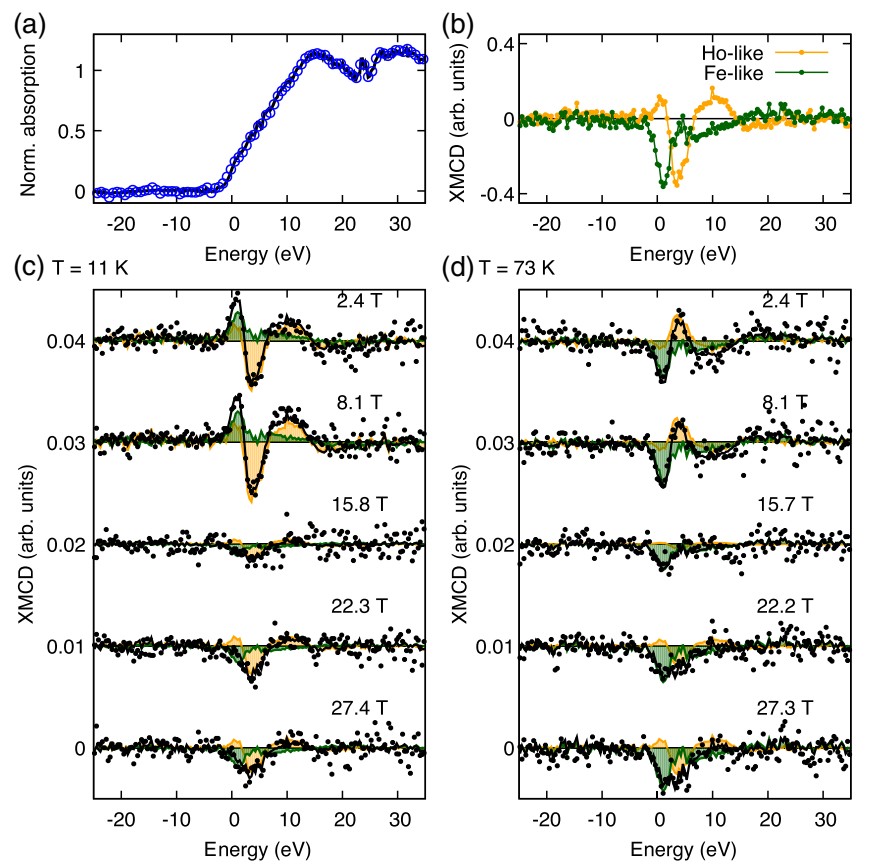

FIG. 2. Fe- $K$-edge absorption and XMCD spectra. (a) Normalized absorption and (b) orthonormal base constructed from the two dominating components representing the Fe and Ho sublattices. XMCD (c) below and (d) above the compensation temperature. Dots, raw data for selected spectra acquired during the falling part of the field pulse; black lines, reconstruction of the data using the spectral components shown as shaded areas. Green, Fe sublattice; orange, Ho sublattice. contribution. For this purpose, we constrain the ratio of the zero-field amplitudes obtained by SVD to the ratios of the corresponding sublattice magnetizations, i.e., $\mathrm{XMCD}_{\mathrm{Fe}}\left(T<T_{\text {comp }}\right) / \mathrm{XMCD}_{\mathrm{Fe}}\left(T>T_{\text {comp }}\right)=$ $M_{\mathrm{Fe}}\left(T<T_{\text {comp }}\right) / M_{\mathrm{Fe}}\left(T>T_{\text {comp }}\right)$.

Figure 2(a) shows the normalized Fe- $K$-edge absorption and Fig. 2(b) the orthonormal base used to reconstruct the original data. We note that the spectral profile of the Fe-like contribution along the [110] direction does not resemble the one for the XMCD signal coming purely from Fe observed for other rare-earth transition-metal intermetallic compounds with nonmagnetic rare-earth compounds in the literature obtained from powder samples [18]. Figures 2(c) and 2(d) show actual XMCD spectra at selected field values above and below the compensation point and the reconstruction using a linear combination of the components. Figures 3(a) and 3(b) show the bulk magnetization and its field derivative above and below the compensation point. At $11 \mathrm{~K}$, a first-order phase transition is observed between 14 and $19.5 \mathrm{~T}$. At $73 \mathrm{~K}$, two weak transitions can be resolved between 5.5 and $6 \mathrm{~T}$ and between 11 and $15 \mathrm{~T}$.

These macroscopic data are to be compared with the field-dependent $\mathrm{Fe}$ and $\mathrm{Ho}$ sublattice magnetizations inferred from the XMCD data. Figures 3(c) and 3(d) show the amplitudes of the Fe- and Ho-like components obtained from the reconstruction of the original data. The following picture is obtained from these field dependences: Below $T_{\text {comp }}$ [Fig. 3(c)] at low field, the Fe and Ho magnetic moments are opposite and remain constant up to the firstorder transition centered around $17 \mathrm{~T}$ and accompanied by large hysteresis of 3-5 T. At the transition, the $\mathrm{Fe}$ magnetization reverses its sign and its projection onto the field direction is reduced to about 25 percent of its lowfield value, whereas the projection of the Ho magnetization
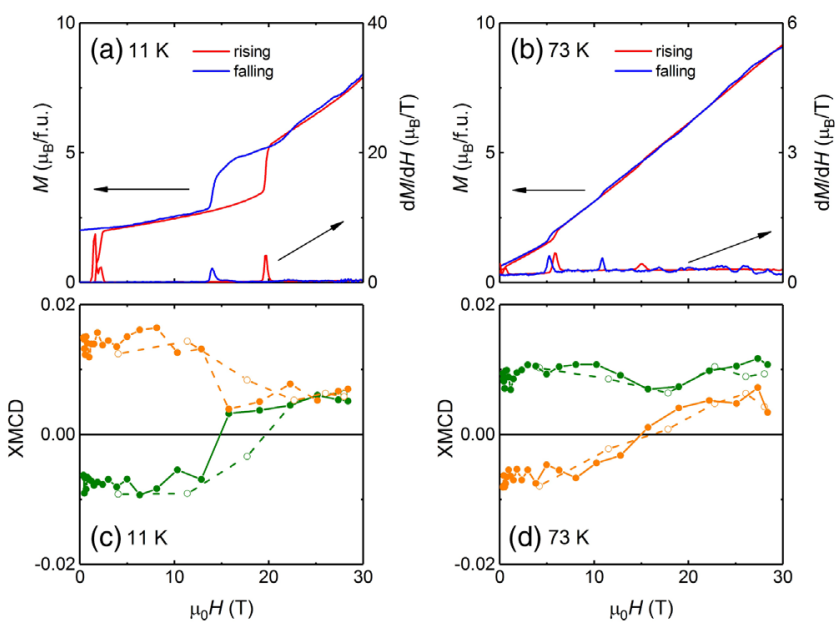

FIG. 3. Bulk magnetization and field derivative of the (a),(b) magnetization and (c),(d) field dependences of the amplitudes of the Fe-like (green) and Ho-like (orange) components below and above the compensation temperature. Open symbols and dashed lines, rising field; full symbols and lines, decreasing field. 
decreases to about 30 percent. Above the transition, both sublattice magnetizations increase monotonically. Above $T_{\text {comp }}$ [Fig. 3(d)], at first sight, the Fe sublattice magnetization exhibits little change around a more or less constant average. The Ho-sublattice magnetization, on the other hand, first remains constant and negative up to about $6 \mathrm{~T}$, where it starts to increase continuously, reversing sign at approximately $16 \mathrm{~T}$ before probably saturating again at a lower absolute value around $27.5 \mathrm{~T}$. Closer inspection of the Fe magnetization indicates two small hysteresis loops opening at around 10 and $27.5 \mathrm{~T}$ that could be concomitant with the beginning and the end of the reversal of the Ho magnetization. At the same time, one might associate a slight dip in the $\mathrm{Fe}$ magnetization with the reversal of the Ho magnetization. The data at the Ho $L_{2,3}$ edges (unpublished) confirm the general picture. A detailed discussion of the XMCD data will be reported elsewhere [34].

In a previous work [10], the field-induced magnetic transition was interpreted as a simultaneous rotation of the $\mathrm{Ho}$ and $\mathrm{Fe}$ magnetic moments from the easy [110] direction to another easy direction in the basal plane, [1-10]. Our $\mathrm{XMCD}$ results seem to confirm this interpretation close to the ground state (at $11 \mathrm{~K}$ ) as the projection of the Ho magnetic moment onto the field direction decreases, whereas the projection of the Fe magnetic moment changes sign at the transition. At $73 \mathrm{~K}$, the situation is more complex, as it is close to the compensation point leading to a large susceptibility, $d M / d H$.

To estimate the in-plane anisotropy constant from our magnetization and the XMCD data, we consider the free energy of a ferrimagnet comprising the energy of the intersublattice exchange, the magnetic anisotropy energy, and the Zeeman energy:

$$
\begin{aligned}
E(\alpha, \phi, B)= & M_{\mathrm{Ho}} M_{\mathrm{Fe}} n_{\mathrm{HoFe}} \cos (\alpha)-K \cos (4 \phi) \\
& -\mu_{0} H\left[M_{\mathrm{Ho}} \sin (\phi)+M_{\mathrm{Fe}} \sin (\alpha+\phi)\right],
\end{aligned}
$$

where $n_{\mathrm{HoFe}}=4 \mathrm{~T} / \mu_{B}$ (at $2 \mathrm{~K}$ ) is the intersublattice exchange parameter [10] and $K$ is the in-plane anisotropy constant. The magnetic anisotropy of the Fe sublattice is neglected here. $\alpha$ is the angle between the Ho and Fe magnetic moments, and $\phi$ is the angle between the Ho magnetic moment and the [110] axis. Since the magnetization vector rotates within the easy basal plane, the uniaxialanisotropy terms are not included in Eq. (1). For each field value, we find equilibrium values of $\alpha$ and $\phi$ by minimizing the free energy (1) with respect to them.

Taking the anisotropy into account allows us to nicely reproduce our experimental data at $11 \mathrm{~K}$ [Fig. 4(a)]. The model reproduces correctly not only the bulk magnetization but also the magnetization of the individual sublattices [Fig. 4(c)]. The field projection of the Ho magnetic moment decreases, whereas that of the $\mathrm{Fe}$ magnetic moment changes sign through the transition. The critical field of the transition depends on $K$ changing from $11 \mathrm{~T}$ for
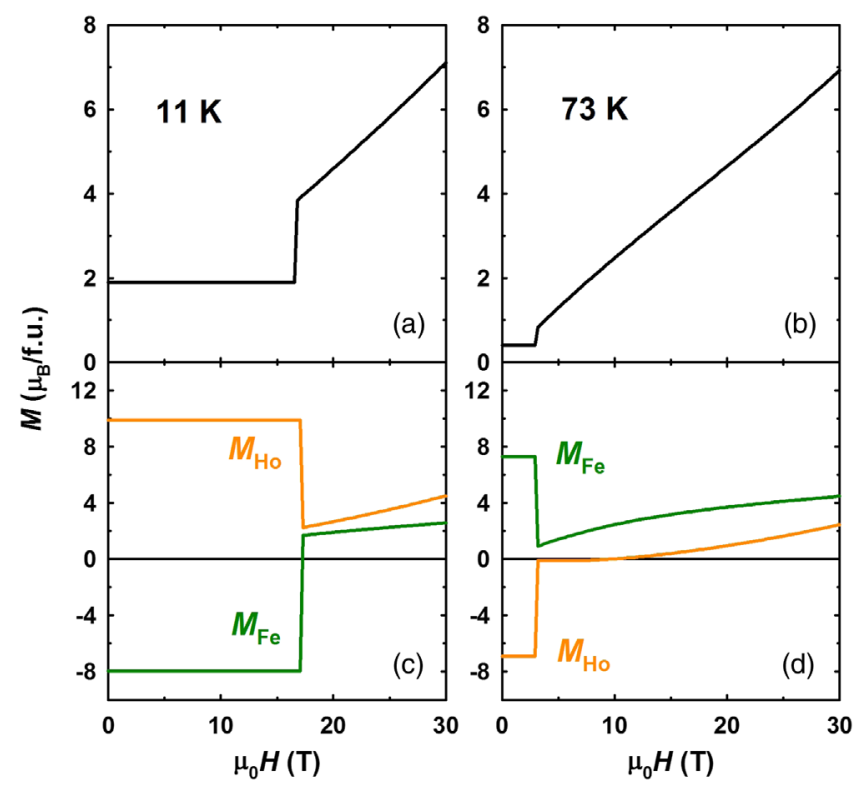

FIG. 4. Field-dependent bulk magnetization at (a) $11 \mathrm{~K}$ and (b) $73 \mathrm{~K}$, and $\mathrm{Ho}$ and $\mathrm{Fe}$ magnetic moments at (c) $11 \mathrm{~K}$ and (d) $73 \mathrm{~K}$ calculated using Eq. (1). At 11 and $73 \mathrm{~K}$, the anisotropy constant was assumed to be 0.5 and $0.25 \mathrm{MJ} / \mathrm{m}^{3}$, respectively.

$K \approx 0.05 \mathrm{MJ} / \mathrm{m}^{3}$ to $17 \mathrm{~T}$ for $K \approx 0.5 \mathrm{MJ} / \mathrm{m}^{3}$. The value of $17 \mathrm{~T}$ corresponds to the center of the transition observed experimentally [Fig. 3(a)]. For higher $K$ values, the transition field hardly becomes larger. Therefore, we can estimate the anisotropy constant within the basal plane to be of the order of $0.5 \mathrm{MJ} / \mathrm{m}^{3}$.

The field-dependent magnetization modeled at $73 \mathrm{~K}$ [Figs. 4(b) and 4(d)] bears two principal differences as compared to our experimental data: The large susceptibility is not reproduced in the low-field region, and only one phase transition is found up to $30 \mathrm{~T}$ in our model. To correct the former, it should be noted that the Ho sublattice is likely not to be rigid above $T_{\text {comp }}$. As the total magnetization is dominated by the Fe sublattice, the applied magnetic field is antiparallel to the molecular field created on the Ho atoms. Therefore, the Ho magnetic moment is reduced by the applied field [see Eq. (1) in the Supplemental Material [18]] and, after passing through zero at $15 \mathrm{~T}$ [Fig. 3(d)], increases along the field direction. By contrast, at $11 \mathrm{~K}$ the applied field is along the molecular field, and the Ho moment is always fully saturated.

In the vicinity of $T_{\text {comp }}$, a ferrimagnet behaves as an antiferromagnet. It has a larger field susceptibility when the magnetic moments of both sublattices are perpendicular to the applied field. For an antiferromagnet with a moderate anisotropy, this state is usually reached through a spin-flop transition. However, a reduction of the Ho moment in the external field stabilizes the orientation of the sublattice magnetization parallel to the field direction. Our estimations show that for $\mathrm{HoFe}_{5} \mathrm{Al}_{7}$ at $73 \mathrm{~K}$ the energies of these two states are close to each other. Therefore, the 
magnetization jumps in Fig. 3(b) are smeared. The XMCD data of Fig. 3(d) show an appreciable reduction of the Fe moment in the field range 5-17 T, which is indicative of the smeared field-induced spin reorientation, at least for a part of the single crystal. This spin reorientation is reminiscent of a spin-flop transition. For very high magnetic fields, the sublattice magnetizations should be oriented parallel to the field direction. For antiferromagnets, this spin configuration is approached continuously without anomalies above the spin flop. In contrast to this behavior, as the magnetic field exceeds $17 \mathrm{~T}$, the $\mathrm{Fe}$ moment of $\mathrm{HoFe}_{5} \mathrm{Al}_{7}$ increases and another small magnetization jump occurs due to the magnetization rotation back towards the applied field.

In conclusion, we investigated the microscopic nature of the spin reorientations underlying the steplike changes in the bulk magnetization of $\mathrm{HoFe}_{5} \mathrm{Al}_{7}$. Near the ground state, we showed that both moments rotate simultaneously from one easy direction to another easy direction in the basal plane in a stepwise manner. We further showed that the Ho moment is no longer rigid near the compensation point, leading to an additional field-induced phase transition as the magnetization rotates back towards the applied field. By use of a simple model, we found that the observed behavior is a general feature of rare-earth transition-metal ferrimagnets with a compensation point when an applied field tunes the balance between antiferromagnetic intersublattice exchange and anisotropy. Our findings provide the first microscopic insight into the magnetization process of a strongly anisotropic ferrimagnet in pulsed magnetic fields.

We acknowledge the attribution of ESRF beam time under proposal HC782. The authors gratefully acknowledge the financial support provided by FRM II to perform the neutron-scattering measurements at the Heinz MaierLeibnitz Zentrum (MLZ), Garching, Germany. We acknowledge the support of the HLD at HZDR, member of the European Magnetic Field Laboratory (EMFL). This work was supported by the Materials Growth and Measurement Laboratory [35], by Grants No. 1603593S, No. 19-00925S, No. 19-07931Y of the Czech Science Foundation, and by Grant No. 18-02-00294 of RFBR. C. S. acknowledges the support of the Helmholtz Association through project-oriented funds.

[1] I. A. Campbell, J. Phys. F 2, L47 (1972).

[2] J. J. M. Franse and R. J. Radwański, in Handbook of Magnetic Materials, Vol. 7, edited by K. H. J. Buschow (Elsevier, Amsterdam, 1993), p. 307.

[3] J. J. M. Franse, F. R. de Boer, P. H. Frings, R. Gersdorf, A. Menovsky, F. A. Muller, R. J. Radwański, and S. Sinnema, Phys. Rev. B 31, 4347 (1985).

[4] R. Ballou, V. M. T. S. Barthem, and D. Gignoux, Physica (Amsterdam) 149B+C, 340 (1988).

[5] J. J. M. Franse, F. E. Kayzel, C. Marquina, R. J. Radwański, and R. Verhoef, J. Alloys Compd. 181, 95 (1992).
[6] S. Yoshii, M. Hagiwara, F. R. de Boer, H. Z. Luo, G. H. Wu, F. M. Yang, and K. Kindo, Phys. Rev. B 75, 214429 (2007).

[7] M. D. Kuz'min, Phys. Rev. B 79, 212405 (2009).

[8] Y. Skourski, M. D. Kuzmin, K. P. Skokov, A. V. Andreev, and J. Wosnitza, Phys. Rev. B 83, 214420 (2011).

[9] D. I. Gorbunov, A. V. Andreev, Y. Skourski, and M. D. Kuzmin, J. Alloys Compd. 553, 358 (2013).

[10] D. I. Gorbunov, S. Yasin, A. V. Andreev, Y. Skourski, Z. Arnold, S. Zherlitsyn, and J. Wosnitza, J. Phys. Condens. Matter 26, 136001 (2014).

[11] W. Kockelmann, W. Schäfer, G. Will, P. Fischer, and J. Gal, J. Alloys Compd. 207-208, 311 (1994).

[12] In Ref. [11], the data for $\mathrm{HoFe}_{5} \mathrm{Al}_{7}$ were evidently interchanged with those of $\mathrm{TmFe}_{5} \mathrm{Al}_{7}$. This follows from the ground-state values of the Ho and Tm magnetic moments reported to be close to 6 and $10 \mu_{B}$, respectively. According to Hunds rules, the highest possible Ho magnetic moment is $10 \mu_{B}$, whereas the Tm magnetic moment cannot exceed $7 \mu_{B}$. Additionally, incorrect anisotropy types in the ground state were given for $\mathrm{HoFe}_{5} \mathrm{Al}_{7}$ (easy axis) and $\mathrm{TmFe}_{5} \mathrm{Al}_{7}$ (easy plane). Our single-crystal magnetization data indicate that the magnetic moments of $\mathrm{HoFe}_{5} \mathrm{Al}_{7}$ lie in the basal plane, whereas those of $\mathrm{TmFe}_{5} \mathrm{Al}_{7}$ are aligned along the [001] axis [13].

[13] D. I. Gorbunov, S. Yasin, A. V. Andreev, Y. Skourski, N. V. Mushnikov, E. V. Rosenfeld, S. Zherlitsyn, and J. Wosnitza, J. Magn. Magn. Mater. 383, 208 (2015).

[14] Heinz Maier-Leibnitz Zentrum, J. Large-Scale Res. Facil. 1, A4 (2015).

[15] V. Petříček, M. Dušek, and L. Palatinus, Z. Kristallogr. 229, 345 (2014).

[16] W. Schäfer, W. Kockelmann, G. Will, P. Fischer, and J. Gal, J. Alloys Compd. 207-208, 316 (1994).

[17] B. J. Campbell, H. T. Stokes, D. E. Tanner, and D. M. Hatch, J. Appl. Crystallogr. 39, 607 (2006).

[18] See Supplemental Material at http://link.aps.org/ supplemental/10.1103/PhysRevLett.122.127205, which includes Refs. [19,20,21], for the refinement of the singlecrystal neutron data, determination of the magnetization of individual sublattices using molecular-field theory, and spectral shape of the Fe-like contribution to the XMCD signal and sensitivity to crystal orientation.

[19] D. I. Gorbunov, A. V. Andreev, S. Daniš, and Pospíšil, J. Alloys Compd. 563, 63 (2013).

[20] R. Boada, C. Piquer, M. A. Laguna-Marco, and J. Chaboy, Phys. Rev. B 82, 052407 (2010).

[21] O. Mathon, F. Baudelet, J. P. Itié, A. Polian, M. d'Astuto, J. C. Chervin, and S. Pascarelli, Phys. Rev. Lett. 93, 255503 (2004).

[22] S. V. Gallego, E. S. Tasci, G. de la Flor, J. M. Perez-Mato, and M. I. Aroyo, J. Appl. Crystallogr. 45, 1236 (2012).

[23] P. van der Linden, O. Mathon, C. Strohm, and M. Sikora, Rev. Sci. Instrum. 79, 075104 (2008).

[24] S. Pascarelli, O. Mathon, T. Mairs, I. Kantor, G. Agostini, C. Strohm, S. Pasternak, F. Perrin, G. Berruyer, P. Chappelet, C. Clavel, and M. C. Dominguez, J. Synchrotron Radiat. 23, 353 (2016).

[25] C. Strohm, F. Perrin, M.-C. Dominguez, J. Headspith, P. van der Linden, and O. Mathon, J. Synchrotron Radiat. 18, 224 (2011). 
[26] J. Chaboy, H. Maruyama, L. M. García, J. Bartolomé, K. Kobayashi, N. Kawamura, A. Marcelli, and L. Bozukov, Phys. Rev. B 54, R15637 (1996).

[27] J. Chaboy, L. M. García, F. Bartolomé, H. Maruyama, A. Marcelli, and L. Bozukov, Phys. Rev. B 57, 13386 (1998).

[28] J. Chaboy, M. A. Laguna-Marco, M. C. Sánchez, H. Maruyama, N. Kawamura, and M. Suzuki, Phys. Rev. B 69, 134421 (2004).

[29] J. Chaboy, L. M. García, and H. Maruyama, Solid State Commun. 107, 317 (1998).
[30] J. Chaboy, L. M. García, F. Bartolomé, A. Marcelli, G. Cibin, H. Maruyama, S. Pizzini, A. Rogalev, J. B. Goedkoop, and J. Goulon, Phys. Rev. B 57, 8424 (1998).

[31] M.-A. Laguna-Marco, J. Chaboy, and C. Piquer, Phys. Rev. B 77, 125132 (2008).

[32] M.-A. Laguna-Marco, J. Chaboy, and H. Maruyama, Phys. Rev. B 72, 094408 (2005).

[33] C. Strohm et al., preceding Letter, Phys. Rev. Lett. 122, 127204 (2019).

[34] C. Strohm et al. (unpublished).

[35] See https://mgml.eu. 